\title{
Evaluating preferences for profiles of GLP-I receptor agonists among injection-naïve type 2 diabetes patients in the UK
}

This article was published in the following Dove Press journal:

Patient Preference and Adherence

9 November 2015

Number of times this article has been viewed

\author{
Heather L Gelhorn' \\ Jiat-Ling Poon' \\ Evan W Davies ${ }^{2}$ \\ Rosirene Paczkowski ${ }^{3}$ \\ Sarah E Curtis ${ }^{3}$ \\ Kristina S Boye ${ }^{3}$ \\ 'Outcomes Research, Evidera, \\ Bethesda, MD, USA; ${ }^{2}$ Outcomes \\ Research, Evidera, London, UK; \\ ${ }^{3}$ Global Patient Outcomes and \\ Real World Evidence, Eli Lilly and \\ Company, Indianapolis, IN, USA
}

Correspondence: Heather L Gelhorn Evidera, 7I0I Wisconsin Avenue, Suite 1400, Bethesda, MD 20814, USA

$\mathrm{Tel}+\mathrm{I} 3032162274$

$\mathrm{Fax}+|30327| 3665$

Email heather.gelhorn@evidera.com
Objective: To use a discrete choice experiment (DCE) to evaluate preferences for the actual treatment features and overall profiles of two injectable glucagon-like peptide-1 receptor agonists (dulaglutide and liraglutide) among patients with type 2 diabetes mellitus (T2DM) in the UK. Methods: In-person interviews were conducted in the UK to administer a DCE to patients with self-reported T2DM, naïve to treatment with injectable medications. The DCE examined six attributes of T2DM treatment each described by two levels: "dosing frequency," "hemoglobin A1c change," "weight change," "type of delivery system," "frequency of nausea," and "frequency of hypoglycemia." Part-worth utilities were estimated using random effects logit models and were used to calculate relative importance (RI) values for each attribute. A chi-square test was used to determine differences in preferences for dulaglutide versus liraglutide profiles.

Results: A total of 243 participants [mean age: 60.5 (standard deviation 10.9) years; $76.1 \%$ male; mean body mass index: 29.8 (standard deviation 5.4 ) $\left.\mathrm{kg} / \mathrm{m}^{2}\right]$ completed the study. RI values for the attributes in rank order were: "dosing frequency" (41.6\%), "type of delivery system" (35.5\%), "frequency of nausea" (10.4\%), "weight change" (5.9\%), "hemoglobin A1c change" (3.6\%), and "frequency of hypoglycemia" (3.0\%). Significantly more participants preferred the dulaglutide profile $(83.1 \%)$ compared with the liraglutide profile $(16.9 \% ; P<0.0001)$.

Conclusion: This study elicited patients' preferences for attributes and levels representing the actual characteristics of two specific glucagon-like peptide- 1 medications. In this context, dosing frequency and type of delivery system were most important, accounting for over 75\% of the RI. While previous studies have identified efficacy as highly important in T2DM medication decisions, this study suggests that when differences in efficacy between medications are small, other treatment features (eg, dosing frequency and delivery system) are of much greater importance to patients.

Keywords: discrete choice experiment, patient preference, type 2 diabetes, GLP-1 receptor agonist

\section{Introduction}

The prevalence of type 2 diabetes mellitus (T2DM) globally is rapidly increasing, precipitated by several important factors including increased life expectancy, an aging population, and lifestyle changes. ${ }^{1}$ The aim of T2DM treatment is to maintain blood glucose levels within acceptable limits, in turn reducing the risk of long-term complications. In most patients, it is common to begin management with lifestyle modifications such as diet, exercise, and weight management. With worsening glucose control the next step is to prescribe oral T2DM drugs. Patients may then progress to injectable therapy, where treatment options include glucagon-like peptide-1 (GLP-1) receptor agonist medications and insulin, if other medications fail to control blood glucose..$^{2,3}$ 
GLP-1 receptor agonists optimize glycemic control by treating hyperglycemia while minimizing or avoiding hypoglycemia, and have acceptable and manageable side effects. ${ }^{4}$ In addition, this class of compounds has also been shown to cause weight loss. ${ }^{5}$ Two examples of GLP-1 receptor agonists include dulaglutide and liraglutide. Both of these compounds are administered as subcutaneous injections, dulaglutide once weekly and liraglutide once daily.

It is important to understand patients' preferences for T2DM medications, and the factors that influence these preferences. Joint recommendations by the European Association for the Study of Diabetes and American Diabetes Association reference the importance of patient's involvement in treatment decisions, ${ }^{2,3}$ stating that:

All treatment decisions, where possible, should be made

in conjunction with the patient, focusing on his/her prefer-

ences, needs, and values. ${ }^{2}$

In order to understand patients' preferences for the clinical and nonclinical features of GLP-1 receptor agonist medications, specifically dulaglutide and liraglutide, a discrete choice experiment (DCE) was conducted.

In a DCE, participants are presented with sets of hypothetical medication choices in order to estimate the relative preferences for different features of the medications. This decision-making approach closely resembles the way individuals consciously or unconsciously make decisions on a daily basis and allows for the determination of the relative importance (RI) of different attributes in determining the overall value of a treatment. The DCE approach was used as it is efficient and appropriate for making comparisons between medication profiles, and can be used to estimate differences in patients' preferences for specific treatment options.

There have been several prior studies examining medication preferences among patients with T2DM. Studies aimed at characterizing patients' overall preferences for the various attributes of T2DM medications have most commonly included efficacy (ie, hemoglobin A1c $\left[\mathrm{HbA}_{1 \mathrm{c}}\right]$ ), hypoglycemia, nausea, and weight change as attributes. ${ }^{6-11}$ Other attributes that have previously been included in T2DM patient preference studies include dosing frequency, urinary tract/genital infections, treatment in case of uncontrolled blood glucose, blood pressure, cardiovascular issues, device storage conditions, pain, injection site reaction, cost, and pill burden. ${ }^{6-12}$

These prior DCE studies focused on identifying which medication characteristics were most important overall for diabetes patients, and have concluded that frequency of hypoglycemia, efficacy (ie, $\mathrm{HbA}_{1 \mathrm{c}}$ change), weight change, and frequency of nausea are generally of greatest importance. ${ }^{6-11}$ However, these prior studies were not designed to specifically examine the RI of the medication characteristics when they reflect the actual differences that are observed between existing medications. For example, while efficacy (ie, $\mathrm{HbA}_{1 \mathrm{c}}$ change, frequency of hypoglycemia, etc) is almost always identified as important, many diabetes products, both oral and injectable, have highly similar efficacy. ${ }^{13-15}$ Thus, although the attribute is important to patients, it may not be the most important consideration when patients and providers are presented with decisions between existing medication options that have highly similar efficacy.

The design of most prior DCEs has often included a range of levels for each attribute that represents the variability observed across an entire class of medications or treatments. As a consequence, the results from studies which employ these general DCE designs reflect the RI of each attribute assuming the maximum variability for each attribute. In real-world treatment contexts, the available medications that physicians and patients may be considering as treatment options are unlikely to reflect the extremes in variability across all attributes. The design of the current study is informative because, when contrasted with results from prior studies, the findings will provide information on whether patients' preferences derived from more general designs are consistent with those designed to investigate a very specific medication comparison. As noted earlier, general patient preference studies in T2DM indicate that efficacy, hypoglycemia, weight change, and frequency of nausea are the primary drivers of patients' medication decisions. The current study will help to elucidate the extent to which general DCE designs provide useful information in predicting patients' preferences when making very specific medication choices. To our knowledge, this is the first study to use this approach. In contrasting the results of the current study with those of previous DCEs, we may also be able to draw some conclusions of the magnitude of differences in each attribute that are important to patients.

To achieve this, the current study was aimed at characterizing patients' preferences for medication attributes specifically in the context of a comparison of the profiles for dulaglutide and liraglutide. As demonstrated by the results of a recent clinical trial (Assessment of Weekly Administration of dulaglutide in Diabetes-6 [AWARD-6]), dulaglutide and liraglutide have medication profiles that are very similar. ${ }^{15}$ In the study, the two medications did not show statistically significant differences on $\mathrm{HbA}_{1 \mathrm{c}}$ change, frequency 
of nausea, or frequency of hypoglycemia. While patients in both groups had significant reduction in weight, patients lost a statistically significantly greater amount of weight on liraglutide. Although the results from the AWARD-6 trial provide information on which characteristics of dulaglutide and liraglutide are statistically different, they do not inform us of the clinical meaningfulness of these differences. DCEs provide an opportunity to characterize how important these characteristics, and their corresponding similarities and differences, are to patients. By using the results from AWARD-6 to inform the specification of the attribute levels for the current study, we were able to ensure that the attribute levels reflected the observed differences between the medications in the population of interest.

In addition to the clinical characteristics that are typically evaluated in diabetes trials, other aspects of T2DM medications such as dosing frequency ${ }^{11,12}$ and the type of medication delivery system ${ }^{16}$ may be important factors in determining patients' preferences. Dulaglutide and liraglutide also differ in their dosing frequency and delivery systems. A priori, it might be expected that patients' preferences would strongly favor the medication that requires less frequent dosing, ${ }^{17-19}$ though it is also conceivable that daily regimens may facilitate higher adherence and may be preferred by patients because they fit better with their daily routines and are easier to remember. Results from Silverman et $\mathrm{al}^{20}$ suggested that patients with osteoporosis did not have a significant preference for daily over weekly dosing, and also showed a trend toward monthly dosing being less preferred than weekly or daily dosing.

The objective of this study was to use a DCE to estimate patients' preferences for the treatment features, safety, and efficacy of two specific GLP-1 receptor agonist medications among patients with T2DM in the UK. Additional objectives included: determining patients' preferences for medication profiles representing dulaglutide versus liraglutide and determining patients' willingness to self-inject medication for the treatment of T2DM.

\section{Methods DCE design}

Attributes were selected for the study based on a review of attributes that are commonly included in other studies of T2DM medications (ie, efficacy, weight change, hypoglycemia, and nausea), ${ }^{6-10}$ and those that are particularly relevant to a comparison of dulaglutide and liraglutide (ie, dosing frequency, type of delivery system) (Table 1). The number of attributes included was consistent with other previously published DCE studies, which typically include between three and seven attributes. ${ }^{21}$ The levels for each of the clinical attributes were based on the results of a head-to-head clinical trial (AWARD-6), which compared dulaglutide $1.5 \mathrm{mg}$ (once weekly) and liraglutide $1.8 \mathrm{mg}$ (once daily), ${ }^{15}$ as well as official instructions for use for the dulaglutide and liraglutide delivery devices. ${ }^{22,23}$

The attributes and levels were tested in a pilot study $(n=52)$ and minor adjustments to clarify the instructions and wording of the attributes and levels were made based on participant feedback. In particular, the attribute " $\mathrm{HbA}_{1 \mathrm{c}}$ change" was renamed "blood sugar $\left(\mathrm{HbA}_{1 \mathrm{c}}\right)$ change" following participants' comments that "blood sugar" was easier for patients to understand. The substantive meaning of the attributes and levels, and the participant inclusion and exclusion criteria were unchanged from the pilot to the main study.

Sample size was estimated using the number of choice pairs (eight) included in the DCE, number of alternatives (two)

Table I Attributes and levels for the discrete choice experiment

\begin{tabular}{|c|c|}
\hline Attribute & Levels \\
\hline Dosing frequency & $\begin{array}{l}\text { - Once a day (365 times per year) })^{1} \\
\text { - Once a week (52 times per year) }{ }^{2}\end{array}$ \\
\hline Blood sugar $\left(\mathrm{HbA}_{\mathrm{Ic}}\right)$ change & $\begin{array}{l}\text { - } 67.9 \% \text { of patients taking the medication reach their desired blood sugar }\left(\mathrm{HbA}_{\mathrm{lc}}\right) \text { goals of less than } 7 \%{ }^{1} \\
\text { - } 68.3 \% \text { of patients taking the medication reach their desired blood sugar }\left(\mathrm{HbA}_{\mathrm{Ic}}\right) \text { goals of less than } 7 \%^{2}\end{array}$ \\
\hline Weight change & $\begin{array}{l}\text { - Patients experience an average weight loss of } 2.90 \mathrm{~kg} \text { after the first } 6 \text { months of medication use }{ }^{2} \\
\text { - Patients experience an average weight loss of } 3.61 \mathrm{~kg} \text { after the first } 6 \text { months of medication use }\end{array}$ \\
\hline Type of delivery system & $\begin{array}{l}\text { - Single-use prefilled pen ready for injection }{ }^{2} \\
\text { - Multidose prefilled pen, used with disposable injection needles, dosage selection required }\end{array}$ \\
\hline Frequency of nausea & $\begin{array}{l}\text { - } 20.4 \% \text { of patients experience nausea anytime in the first } 6 \text { months of treatment }{ }^{2} \\
\text { - } 18.0 \% \text { of patients experience nausea anytime in the first } 6 \text { months of treatment }{ }^{1}\end{array}$ \\
\hline $\begin{array}{l}\text { Frequency of low blood sugar } \\
\text { (hypoglycemia) }\end{array}$ & $\begin{array}{l}\text { - On average, a patient would experience one low blood sugar (hypoglycemia) event every } 2 \text { years }{ }^{1} \\
\text { - On average, a patient would experience one low blood sugar (hypoglycemia) event every } 3 \text { years }^{2}\end{array}$ \\
\hline
\end{tabular}

Notes: 'Level representing liraglutide. 'Level representing dulaglutide.

Abbreviation: $\mathrm{HbA}_{1 \mathrm{c}}$, hemoglobin $\mathrm{A}_{\mathrm{Ic}}$. 
per choice pair, and maximum number of levels (two) for any one attribute. Based on these study parameters, the minimum recommended number of participants required for this study, and to allow for a sufficient number of participants for the planned subgroup analyses, a sample size of 260 patients was targeted. ${ }^{24}$ The choice sets were developed using SAS Version 9.4 (SAS Institute Inc., Cary, NC, USA) based on a D-efficiency criterion. ${ }^{25}$

\section{Participants}

Participants were recruited via newspaper advertisements and Internet classified advertisements in four locations in the UK: London, Edinburgh, Birmingham, and Cardiff. Interested participants who responded to the advertisement were screened via telephone to assess eligibility. To be eligible for the study, participants had to meet the following eligibility criteria: male or female $\geq 18$ years of age; diagnosed with T2DM; taking at least one oral T2DM medication; able to read and understand English; a resident of the UK; and willing and able to provide written informed consent. Participants were excluded if they had type 1 diabetes or gestational diabetes; T2DM that is currently treated only with diet and exercise; previously been treated with injectable medication for any medical condition including T2DM; or previously administered an injectable medication to another person for any medical condition including T2DM.

In order to confirm their T2DM diagnosis, participants brought their T2DM prescription medication to the interview or described their diagnosis process. All participants provided informed consent prior to participating in the study and the study protocol was approved by an independent ethical review board (Ethical \& Independent Review Services: November 6, 2014).

\section{Study procedures}

The study consisted of both pilot (September 2014-October 2014) and main study (December 2014-February 2015) components. The pilot study was aimed at assessing the validity of the DCE task and the feasibility of recruitment and in-person implementation. Surveys were administered in-person by trained moderators, though participants selfcompleted the survey questions.

The survey consisted of five sections administered in the following order: 1) a question determining participants' willingness to self-inject medication for T2DM; 2) a description of the DCE portion of the study; 3) the DCE choice task; 4) questions asking about participants' willingness to self-inject a product with profiles representing dulaglutide and liraglutide, respectively; and 5) a sociodemographic and clinical information questionnaire.

Prior to completing the DCE, participants read a brief description of the purpose of the study. This section indicated to the participants that they would be presented with pairs of hypothetical medications and would be asked which of the medications they preferred. The participants reviewed the descriptions of all the medication attributes and levels. Participants then watched two videos that demonstrated the use of devices representing those that are available in the UK for administration of dulaglutide and liraglutide, respectively. $^{22,23}$ The order of presentation of the videos describing the devices was varied by using a block design. No product names were mentioned anywhere in the videos or survey.

The DCE consisted of choices between two hypothetical medications each described by one level for each of the six attributes. For each pair, participants were asked to indicate which medication they preferred. Participants completed ten medication choice pairs. One of the choice pairs presented to each participant was a fixed choice with one clearly correct option. The fixed choice was used to ensure that participants understood the discrete choice format and attended appropriately to the task. Any participants who responded incorrectly to the fixed choice question were excluded from the analysis. Additionally, one of the medication choices presented the medication profiles for dulaglutide and liraglutide, respectively. This choice was embedded within the DCE design and was indistinguishable to participants from the other DCE choices. Participants' responses to this question were used to determine their direct preference for the dulaglutide profile compared with the liraglutide profile, but were not used in estimating the results of the DCE.

Following completion of the DCE survey, participants were asked to answer two questions about their willingness to self-inject medication profiles representing dulaglutide and liraglutide, and to complete a brief questionnaire on demographic and clinical characteristics.

\section{Analyses}

Data from the pilot and main study were pooled for the analyses; this decision was made a priori. SAS Version 9.4 (SAS Institute Inc.) was used to conduct the analyses. Descriptive statistics (frequencies, means, and standard deviations [SD]) were reported for sociodemographic and self-reported clinical variables, and for supplementary 
variables on willingness to use injectable medications and willingness to use medications represented by the dulaglutide and liraglutide profiles. The DCE responses were analyzed using a random effects logit regression model. ${ }^{25}$

To analyze the results of the DCE, part-worth utility estimates were computed for the overall sample, and by age and sex subgroups. Part-worth utility values provide information on the extent to which participants prefer each level of an attribute, and were scaled within each attribute to have a mean of 0 . A positive value indicates that the attribute level is preferred, while negative values indicate a preference for other levels of the attribute. Larger part-worth utility values indicate a higher degree of preference. The part-worth utility values or preference weights reflect the strength of patients' preferences for each of the medication attributes and differ conceptually from utility values which are estimated to calculate quality-adjusted life years.

The RI of each attribute was calculated by summing the range of part-worth utility values for all attributes (ie, the largest minus the smallest part-worth utility values within each attribute), yielding the overall utility value, and then by dividing each individual attribute's utility range by the overall utility value (Table 3 for example calculation). ${ }^{24}$ The RI of each attribute is expressed as a percentage and reflects the proportion of the variance in the overall medication decision that is accounted for by each attribute.

Regression models with interaction effects were used to test for significant differences in preferences for the levels of each attribute across age ( $<60$ years vs $\geq 60$ years) and sex subgroups. Chi-square tests were used to determine whether there were significant differences in the RI of the attributes across age and sex subgroups. The fixed choice question and the question comparing the dulaglutide and liraglutide profiles were not included in the DCE analysis.

\section{Results}

\section{Sample characteristics}

A total of 476 participants were screened with 257 (54.0\%) eligible to participate in the study (Figure 1). Of those deemed ineligible ( $\mathrm{n}=219)$, the top three reasons for ineligibility were: 1) currently treated with oral and injectable medications for T2DM (29.7\%); 2) participants had previously used an injection device (24.7\%); and 3 ) participants were currently treated with injectable medications for T2DM (12.3\%). A total of 254 (98.8\%) participants completed the survey, 11 participants $(4.3 \%)$ gave an incorrect response on the fixed-choice question and their data were excluded from the final analytical sample $(\mathrm{N}=243)$.
The demographic and self-reported clinical characteristics of the participants are presented in Table 2. The mean age of participants was 60.5 (SD 10.9) years, and the sample was $76.1 \%$ male. The sex distribution of the eligible participants was similar to the distribution among those who were screened $(\sim 71.0 \%$ male). The majority of the participants were White $(72.0 \%)$, and the mean body mass index was 29.8 (SD 5.4) (Table 2). Most of the participants reported taking one oral medication (44.9\%) to treat their T2DM, and most had been diagnosed with T2DM between 1 and 5 years $(35.8 \%)$ or between 5 and 10 years $(34.6 \%)$.

Table 2 Self-reported sociodemographic and clinical characteristics

\begin{tabular}{|c|c|}
\hline & Overall sample $\mathbf{N}=\mathbf{2 4 3}$ \\
\hline \multicolumn{2}{|l|}{ Sex n (\%) } \\
\hline Male & $185(76.1)$ \\
\hline Female & $58(23.9)$ \\
\hline Age in years, mean (SD) [range] & $60.5(10.9)[21-88]$ \\
\hline \multicolumn{2}{|l|}{ Age group n (\%) } \\
\hline $18-29$ & $2(0.8)$ \\
\hline $30-39$ & $5(2.1)$ \\
\hline $40-49$ & $30(12.3)$ \\
\hline $50-64$ & $113(46.5)$ \\
\hline $65+$ & $93(38.3)$ \\
\hline \multicolumn{2}{|l|}{ Race/ethnicity n (\%) } \\
\hline White & $175(72.0)$ \\
\hline Mixed/multiple ethnic groups & $7(2.9)$ \\
\hline Asian or Asian-British & $37(15.2)$ \\
\hline Black/African/Caribbean/Black British & $23(9.5)$ \\
\hline Other & $\mathrm{I}(0.4)$ \\
\hline \multicolumn{2}{|l|}{ Employment status n (\%) } \\
\hline Full-time & $67(27.7)$ \\
\hline Part-time & $31(12.8)$ \\
\hline Homemaker/housewife & $5(2.1)$ \\
\hline Student & $3(1.2)$ \\
\hline Unemployed & $26(10.7)$ \\
\hline Retired & $102(42.1)$ \\
\hline Disabled & $8(3.3)$ \\
\hline \multicolumn{2}{|l|}{ Education n (\%) } \\
\hline No formal qualifications & $30(12.4)$ \\
\hline GCSE/‘O' levels or equivalent & $43(17.8)$ \\
\hline 'A' levels or equivalent & $48(19.8)$ \\
\hline Vocational/work-based qualifications & $48(19.8)$ \\
\hline University degree & $40(16.5)$ \\
\hline Postgraduate degree & $33(13.6)$ \\
\hline \multicolumn{2}{|l|}{ Household income $\mathrm{n}(\%)$} \\
\hline Less than $£ 5,200$ & $8(3.3)$ \\
\hline$£ 5,200$ to $£ 10,399$ & $16(6.6)$ \\
\hline$£ \mid 0,400$ to $£ \mid 5,599$ & $25(10.3)$ \\
\hline$£ 15,600$ to $£ 20,799$ & $28(11.5)$ \\
\hline$£ 20,800$ to $£ 25,999$ & $16(6.6)$ \\
\hline$£ 26,000$ to $£ 31,199$ & $22(9.1)$ \\
\hline$£ 31,200$ to $£ 36,399$ & $17(7.0)$ \\
\hline$£ 36,400$ to $£ 51,999$ & $23(9.5)$ \\
\hline$£ 52,000$ or more & $26(10.7)$ \\
\hline Missing & $62(25.5)$ \\
\hline
\end{tabular}


Table 2 (Continued)

\begin{tabular}{|c|c|}
\hline & Overall sample $\mathbf{N}=\mathbf{2 4 3}$ \\
\hline Height (cm), mean (SD) [range] & $172.5(9.7)[150-196]$ \\
\hline Weight (kg), mean (SD) [range] & $88.7(17.3)[48-162]$ \\
\hline BMI $\left(\mathrm{kg} / \mathrm{m}^{2}\right)$, mean $(\mathrm{SD})$ [range] & $29.8(5.4)[18-52]$ \\
\hline \multicolumn{2}{|l|}{ Duration of T2DM n (\%) } \\
\hline Less than I year & $14(5.8)$ \\
\hline More than I year and up to 5 years & $87(35.8)$ \\
\hline More than 5 years and up to 10 years & $84(34.6)$ \\
\hline More than 10 years & $58(23.9)$ \\
\hline \multicolumn{2}{|l|}{ Current T2DM treatment n (\%) } \\
\hline One oral diabetes medication only & $109(44.9)$ \\
\hline Two oral diabetes medication only & $75(30.9)$ \\
\hline Three or more oral diabetes medication & $59(24.3)$ \\
\hline \multicolumn{2}{|l|}{ Current $\mathrm{HbA}_{\mathrm{Ic}}$ level $\mathrm{n}(\%)$} \\
\hline Below 53 mmol/mol (below 7\%) & $70(28.8)$ \\
\hline $\begin{array}{l}\text { Between } 54 \text { and } 64 \mathrm{mmol} / \mathrm{mol} \\
\text { (between } 7.1 \% \text { and } 8 \% \text { ) }\end{array}$ & $62(25.5)$ \\
\hline $\begin{array}{l}\text { Between } 65 \text { and } 75 \mathrm{mmol} / \mathrm{mol} \\
\text { (between } 8.1 \% \text { and } 9 \% \text { ) }\end{array}$ & $27(11.1)$ \\
\hline $\begin{array}{l}\text { Higher than } 75 \mathrm{mmol} / \mathrm{mol} \\
\text { (higher than } 9 \% \text { ) }\end{array}$ & $16(6.6)$ \\
\hline Do not know & $68(28.0)$ \\
\hline
\end{tabular}

Abbreviations: $\mathrm{BMI}$, body mass index; $\mathrm{HbA}_{\mathrm{Ic}}$, hemoglobin $\mathrm{A}_{\mathrm{lc}} ; \mathrm{SD}$, standard deviation; T2DM, type 2 diabetes mellitus.

\section{Results of the DCE}

\section{Preferences in the overall sample}

In the overall sample, the most important attributes were "dosing frequency" (RI=41.6\%) and "type of delivery system" (RI=35.5\%) (Table 3). The remaining attributes "frequency of nausea" (RI=10.4\%), "weight change" ( $\mathrm{RI}=5.9 \%)$, "HbA $\mathrm{Hc}_{1 \mathrm{c}}$ change" ( $\left.\mathrm{RI}=3.6 \%\right)$, and "frequency of hypoglycemia" (RI=3.0\%) were of minor importance. A priori, it was expected that participants would indicate preferences for less frequent dosing, a greater percentage of patients at $\mathrm{HbA}_{1 \mathrm{c}}$ goal, greater weight loss, less nausea, and less frequent hypoglycemia. The results reflected preferences in the expected direction for each of these attributes, providing support for the validity of the study (Table 3; Figure 2). Participants also indicated a preference toward the single-use prefilled pen delivery system. There were significant preferences for the different levels of all attributes (all $P<0.05$ ), except for frequency of hypoglycemia.

\section{Patient preferences by sex}

The preferences of the participants with T2DM were examined by sex (Figure 3). "Dosing frequency" (males, RI=47.0\%; females, RI=25.4\%) and "type of delivery system" (males, $\mathrm{RI}=33.9 \%$; females, $\mathrm{RI}=40.6 \%$ ) had the highest RI. The RI of these attributes differed significantly between males and females with "dosing frequency" more important to males and "type of delivery system" more important to females (both $P<0.0001)$. There were also significant differences between sexes in the strength of their preferences for the levels of

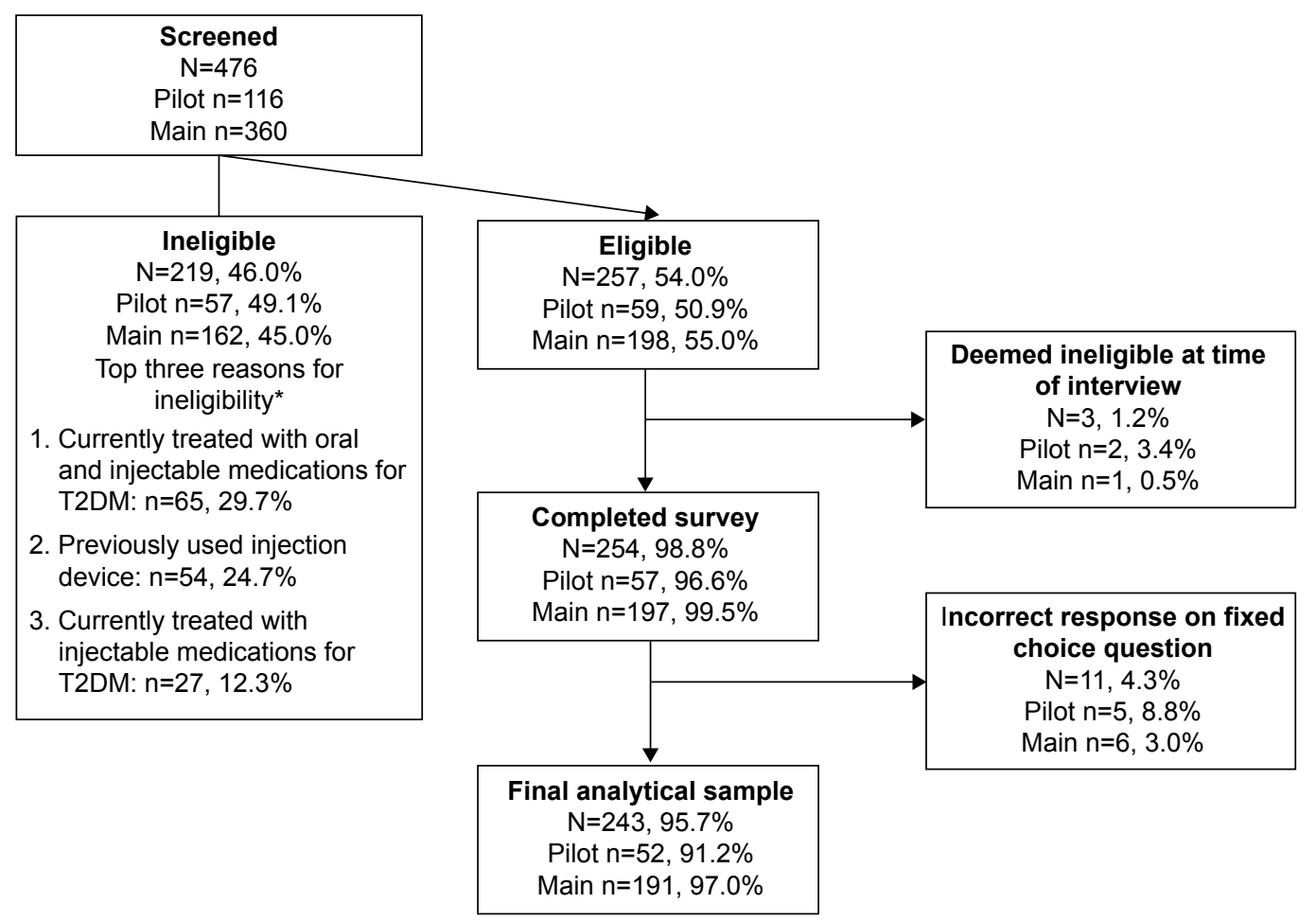

Figure I Participant disposition.

Note: *Excludes participants unwilling/unable to travel to interview location, no shows, and cancellations.

Abbreviation: T2DM, type 2 diabetes mellitus. 
Table 3 Part-worth utilities, RI, and rankings of attributes in overall sample

\begin{tabular}{|c|c|c|c|c|c|c|}
\hline Parameter & Attribute levels & $\begin{array}{l}\text { Part-worth utility } \\
\text { estimate (SE)' }\end{array}$ & $P$-value ${ }^{2}$ & $\begin{array}{l}\text { Overall part-worth } \\
\text { utility value }\end{array}$ & $\mathbf{R} \mathbf{I}^{4}(\%)$ & Rank \\
\hline \multirow[t]{2}{*}{ Dosing frequency } & Once a day (365 times per year) & $-0.46 \mathrm{I}(0.042)$ & $<0.0001$ & 0.92 & 41.6 & I \\
\hline & Once a week (52 times per year) & $0.46 \mathrm{I}(0.042)$ & $<0.0001$ & & & \\
\hline \multirow[t]{2}{*}{ Type of delivery system } & Multidose prefilled pen & $-0.393(0.042)$ & $<0.0001$ & 0.79 & 35.5 & 2 \\
\hline & Single-use prefilled pen & $0.393(0.042)$ & $<0.0001$ & & & \\
\hline \multirow[t]{2}{*}{ Frequency of nausea } & $20.4 \%$ experience nausea & $-0.115(0.022)$ & $<0.0001$ & 0.23 & 10.4 & 3 \\
\hline & $18.0 \%$ experience nausea & $0.115(0.022)$ & $<0.0001$ & & & \\
\hline \multirow[t]{2}{*}{ Weight change } & $2.90 \mathrm{~kg}$ weight loss & $-0.066(0.022)$ & 0.0023 & 0.13 & 5.9 & 4 \\
\hline & $3.61 \mathrm{~kg}$ weight loss & $0.066(0.022)$ & 0.0023 & & & \\
\hline \multirow[t]{2}{*}{ Blood sugar $\left(\mathrm{HbA}_{\mathrm{lc}}\right)$ change } & $67.9 \%$ at goal & $-0.040(0.015)$ & 0.0095 & 0.08 & 3.6 & 5 \\
\hline & $68.3 \%$ at goal & $0.040(0.015)$ & 0.0095 & & & \\
\hline Frequency of low blood sugar & Once every 2 years & $-0.033(0.021)$ & 0.1283 & 0.07 & 3.0 & 6 \\
\hline events (hypoglycemia) & Once every 3 years & $0.033(0.021)$ & 0.1283 & & & \\
\hline
\end{tabular}

Notes: 'Part-worth utility values provide information on the extent to which participants prefer each level of an attribute and are scaled within each attribute to have a mean of 0 . A positive part-worth utility value indicates that the attribute level is preferred, while negative values indicate a preference for other levels of the attribute. ${ }^{2}$ The $P$-values indicate whether the part-worth utility value differs significantly from $0 .{ }^{3}$ The overall utility values represent the range of utility values within each attribute. ${ }^{4} \mathrm{RI}$ for each attribute $=\frac{\text { Overall utility value for each attribute }}{\text { Total utility value }}$, where total utility value $=$ sum of overall utility values across all attributes.

Abbreviations: $\mathrm{HbA}_{\mathrm{Ic}}$, hemoglobin $\mathrm{A}_{\mathrm{Ic}}$; $\mathrm{RI}$, relative importance; $\mathrm{SE}$, standard error.

the "dosing frequency" $(P=0.0298)$ and "weight change" $(P=0.0218)$ attributes. While both sexes preferred less frequent dosing and greater weight loss, males had a significantly stronger preference for once a week dosing, and females had a significantly stronger preference for greater weight loss.

\section{Patient preferences by age group}

The preferences of the participants with T2DM were also examined by age groups (Figure 4). A cut-off of 60 years was used based on the age distribution of the sample. The RI of the attributes was equivalent across the age groups for all attributes except "frequency of hypoglycemia," which was significantly more important to older participants (RI $=0.3 \%$ for $<60$ years; $\mathrm{RI}=5.5 \%$ for $\geq 60$ years; $P=0.0108$ ). There were also significant differences between age groups in the strength of their preferences for levels of two attributes: "dosing frequency" $(P=0.0367)$ and "HbA $1 \mathrm{c}$ change" $(P=0.0354)$. Participants less than 60 years old had a significantly stronger preference for less frequent dosing and for treatments where a larger proportion of patients reached their $\mathrm{HbA}_{1 \mathrm{c}}$ goals.

\section{Preferences for dulaglutide versus liraglutide profiles}

One of the choice pairs embedded among the other DCE questions consisted of two medication profiles representing dulaglutide and liraglutide, respectively. Participants' responses to this question were not included in the estimation of RI but were used to determine direct preference for the dulaglutide profile versus the liraglutide profile. The majority of participants $(83.1 \%$ ) preferred the dulaglutide profile, while $16.9 \%$ preferred liraglutide $(P<0.0001)$. There were no statistical differences between sex or age groups in preferences for the profiles of dulaglutide versus liraglutide.

\section{Willingness to take and self-inject T2DM treatments} Prior to completing the DCE exercise, 92 (37.9\%) participants said that they would be willing to take a diabetes medication that required an injection for each dose, while 94 (38.7\%) participants said that they would not be willing to take a medication that required an injection for each dose. The remaining participants answered either "neutral" or "do not know." The majority of participants $(65.8 \%)$ said that they would prefer to self-inject rather than have someone else inject for them.

After viewing the videos and completing the DCE, $77.0 \%$ of participants said that they were willing to take the medication represented by the dulaglutide profile (somewhat willing, $39.1 \%$; very willing, $37.9 \%$ ). In contrast, $30.5 \%$ of participants were willing to take the medication represented by the liraglutide profile (somewhat willing, $17.7 \%$; very willing, $12.8 \%)(P<0.0001)$.

\section{Discussion}

In this study, patients' preferences for attributes and levels representing the actual characteristics of two specific GLP-1 receptor agonist medications were elicited. These results are important because they are not the same as those from prior studies of T2DM patient preference studies with more general designs. The previous studies with general DCE designs have concluded that frequency of hypoglycemia, 


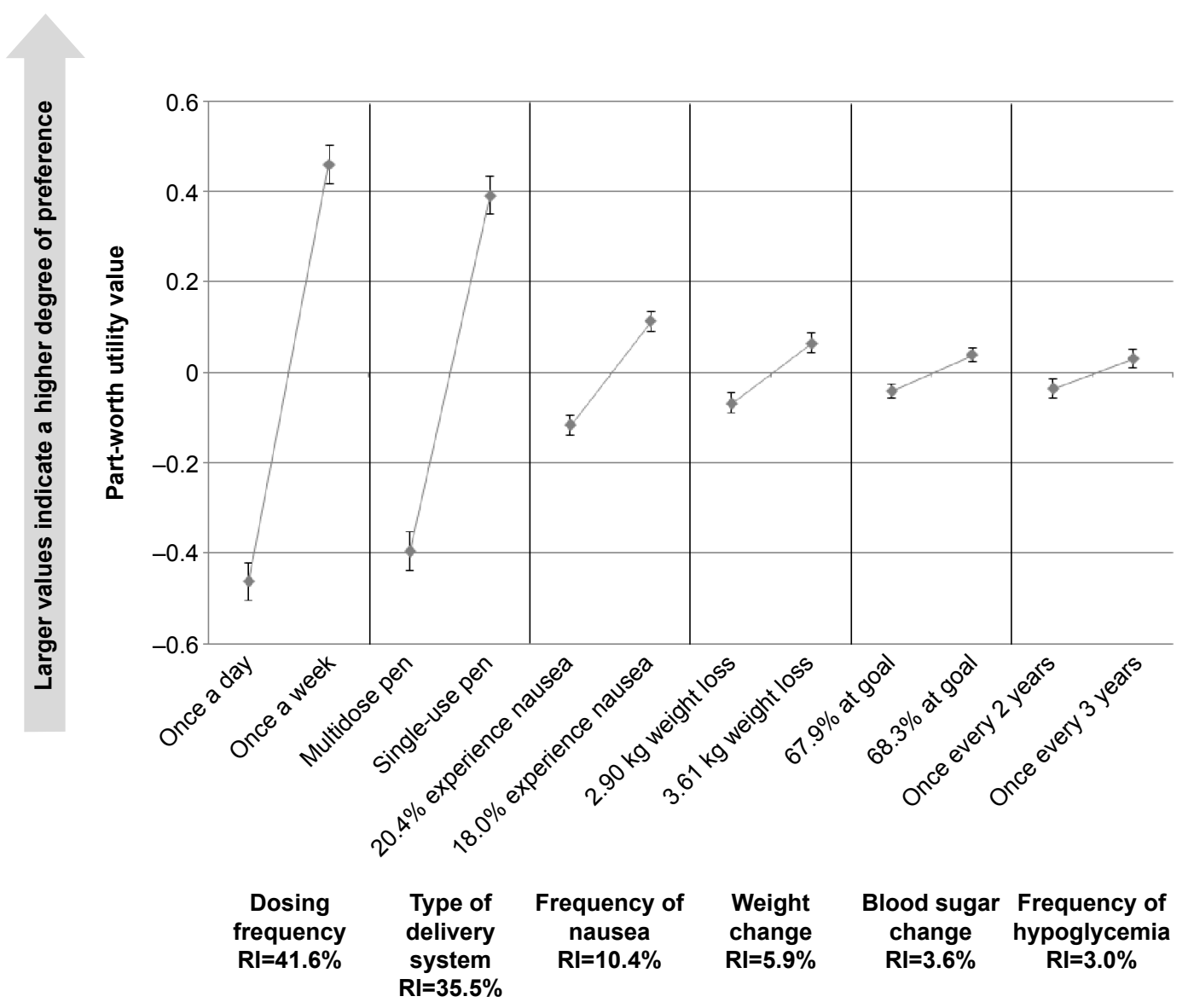

Decreasing order of RI

Figure 2 Relative importance of attributes in overall sample. Abbreviation: RI, relative importance.

efficacy (ie, $\mathrm{HbA}_{1 \mathrm{c}}$ change), weight change, and frequency of nausea are generally of greatest importance. ${ }^{6-11}$ In contrast, the current study, with a very specific medication comparison dictating the design, suggests that dosing frequency and type of delivery system are of greatest importance to patients in this context.

The conflicting results highlight the importance of the research design and context in interpreting the results of DCE studies, and the different information provided by each DCE design option. In a more general design, the results inform us of patients' preferences broadly considered across all of the included attributes and the full range of variability. This likely provides the best information on "what is important to patients overall." In contrast, the current study, with a design that includes a very specific medication comparison, tells us what might drive patients' preferences in a very specific decision context. The discrepancy underscores the importance of the study context in the interpretation of all
DCE studies. This finding is an area for future research, and should be replicated with other medication comparisons and in other disease areas. For now, the results suggest that we should be cautious about taking the conclusions of studies with general DCE designs and applying them to specific medication comparisons. In addition, we should be cautious about using the conclusions of DCEs that examine specific medication comparisons and assuming they provide information on which attributes are most important in a broader context. The results of the general DCEs for example, might help to value and identify attributes that pharmaceutical research and development teams should target for improvement with future medications. In contrast, the results of the more specific DCEs may help clinicians understand which features of existing medications are most attractive to patients currently seeking treatment.

In the context of this specific study, when presented with medication choices representing the characteristics of the 


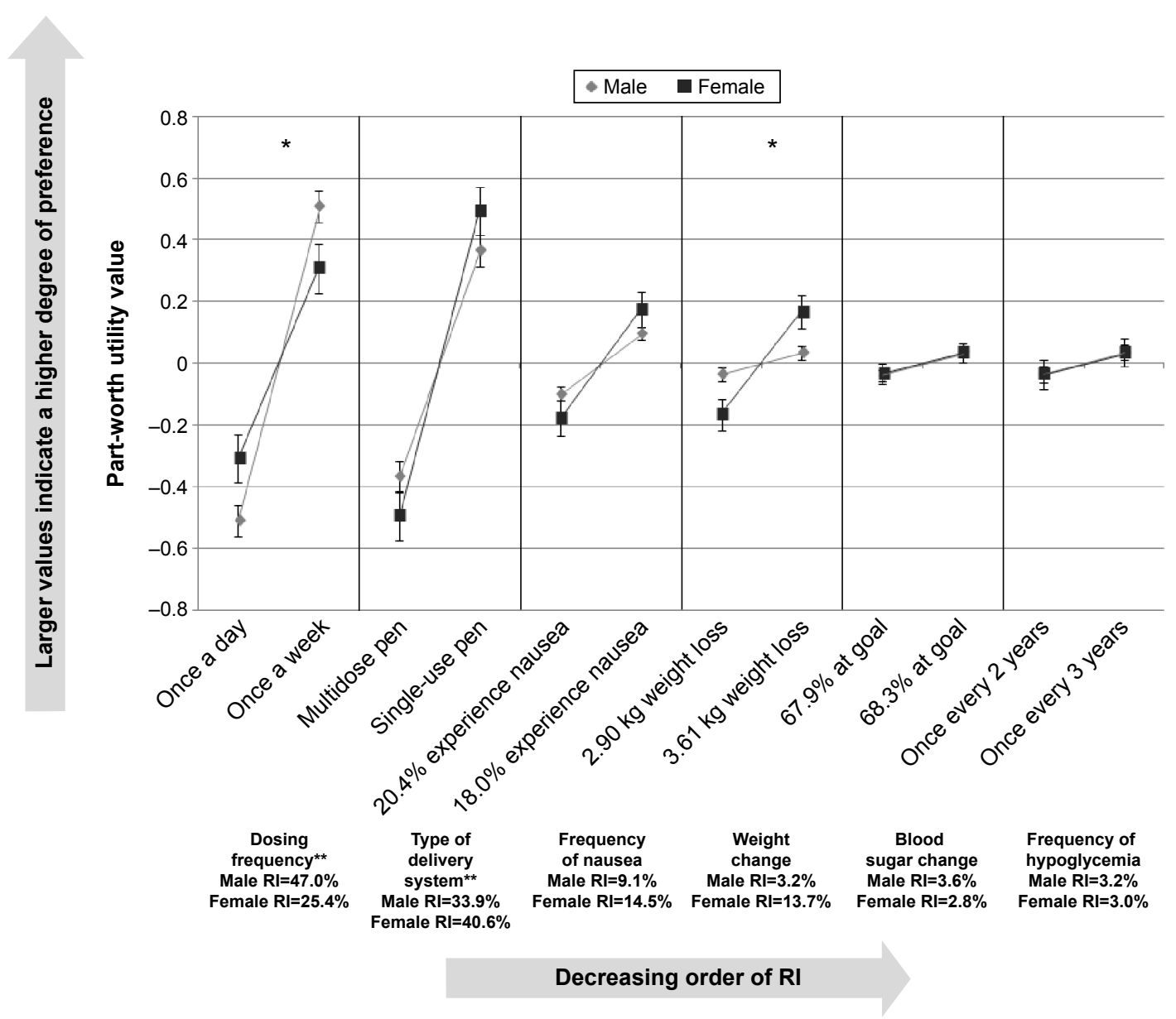

Figure 3 Relative importance of attributes by sex.

Notes: *Statistically significant difference between sexes in preference for levels of the attribute $(P<0.05)$. **Statistically significant difference in the relative importance of each attribute across sex $(P<0.000 \mathrm{I})$.

Abbreviation: RI, relative importance.

available treatment options from the two GLP-1 receptor agonist medications, the most important factors in patients' decisions in rank order were: "dosing frequency," "type of delivery device," "frequency of nausea," "weight change," "HbA $\mathrm{Ac}_{1 \mathrm{c}}$ change," and "frequency of hypoglycemia." Consistent with the results from the DCE portion of the study, when provided with a direct comparison of the medication profiles, the vast majority of patients preferred the medication profile representing the characteristics of dulaglutide $(83.1 \%)$ over the profile representing the characteristics of liraglutide (16.9\%). Also consistent with the findings of the DCE, a much larger proportion of the participants (77.0\%) were willing to take the medication represented by the dulaglutide profile, while less than one-third of the participants $(30.5 \%)$ were willing to take the medication represented by the liraglutide profile.

Previous studies of patients' preferences for attributes of T2DM medications have found glucose control $\left(\mathrm{HbA}_{1 \mathrm{c}}\right.$ change and hypoglycemia) and weight change to be attributes that are highly important in influencing patients' T2DM medication decisions. ${ }^{6-9}$ However, in real-life treatment contexts these quantitative attributes do not always differ between the actual medication options that patients and physicians may be considering. In such cases, the results of this study suggests that when the differences in glucose control between medications is small, other treatment features that may be more qualitative in nature such as dosing frequency and type of delivery system are of much greater importance to patients.

The findings from the current study of GLP-1 receptor agonist treatments are consistent with previous research into patients' preferences for nonclinical features (eg, "dosing frequency," "type of delivery system," "needle size," "pain," "storage conditions," "injection site reaction"), which found that dosing frequency was most important to patients in their T2DM medication decisions in that specific 


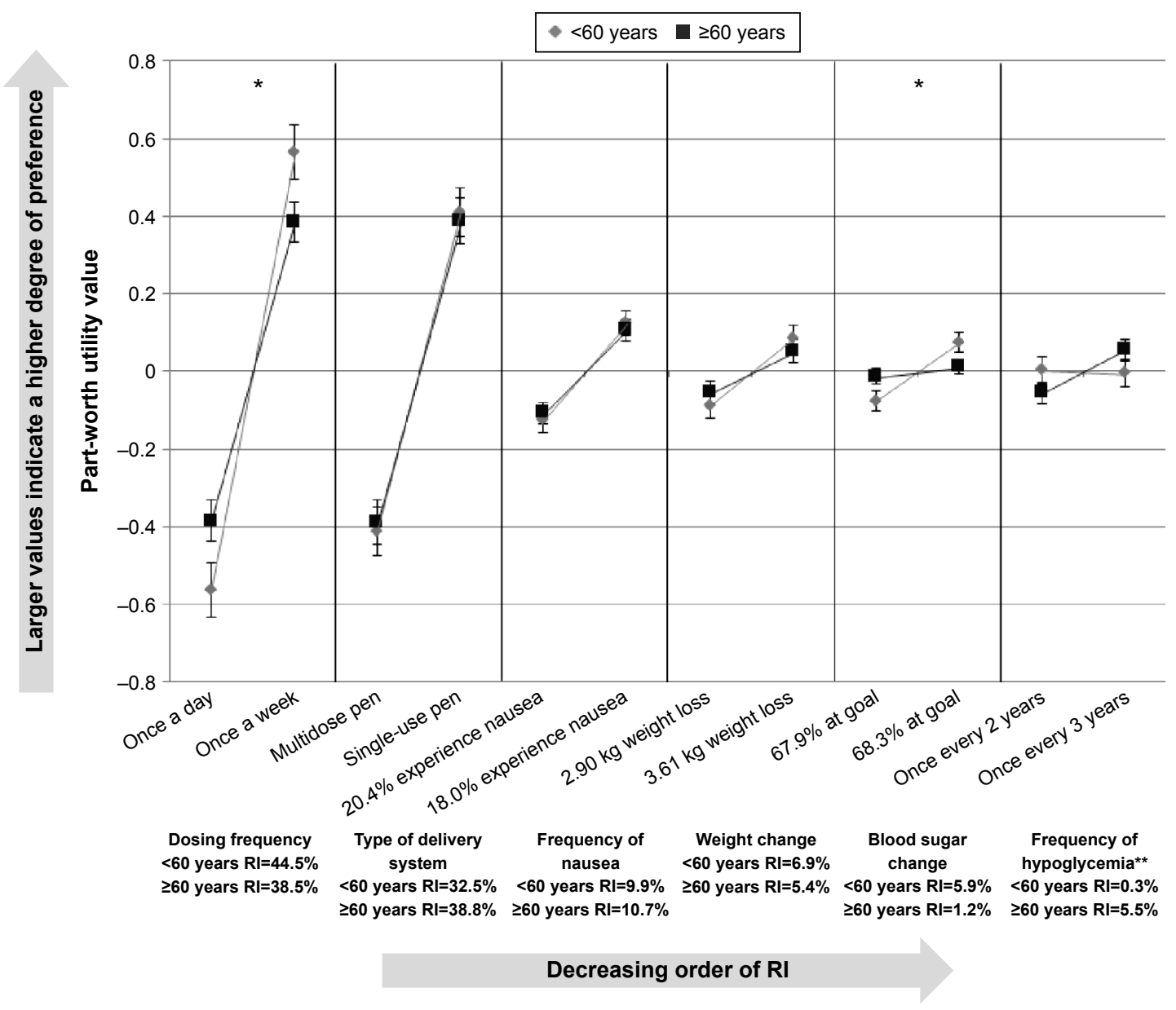

Figure 4 Relative importance of attributes by age group ( $<60$ years vs $\geq 60$ years).

Notes: *Statistically significant difference between age groups in preference for levels of the attribute $(P<0.05)$. **Statistically significant difference in the relative importance of each attribute across age groups $(P=0.0108)$.

Abbreviation: RI, relative importance.

study context. ${ }^{12}$ Unlike some previous studies, this study did not include "needle size," "pain," "injection site reaction," or "cost" as attributes. We selected the attributes which were most commonly included in previous DCE studies in T2DM and thus did not include the first three. While there are various side effects and adverse events associated with GLP-1 medication, those we selected for the current study were the most common and showed the greatest variability between the two medications. Cost was excluded as an attribute because this study was conducted in the UK, where patients do not bear the cost of treatment directly. Had the cost attribute been included, it would have favored dulaglutide. ${ }^{26}$ In addition, it was necessary to limit the number of attributes so as to avoid excessive cognitive burden associated with presenting participants with too many attributes.

The results of the comparison between preferences of males versus females is consistent with previous research, which also found that among patients with T2DM in the UK, weight loss was significantly more important to females than males. ${ }^{27}$ In the current study, it is interesting to note that the RI of this attribute was relatively small compared with dosing frequency and type of delivery system attributes, among both males and females. This suggests that although weight loss may be of greater concern to females in this particular decision context, it remains secondary to considerations related to dosing frequency and type of delivery system. There were also significant differences in the magnitude of the preferences for the dosing frequency, with males having a significantly stronger preference for the less frequent dosing. Among females, the type of delivery system had a higher RI than among males, however, for both sexes, the single-use pen was strongly preferred. Despite these differences across sex in terms of the magnitude and nature of the preferences, the direct comparison of the two medication profiles representing dulaglutide and liraglutide, respectively, did not differ 
significantly by sex, with a strong preference for the profile representing dulaglutide among patients of both sexes.

There were several reasons that participants were excluded from the study if they had any experience with injectable medications. Based on the European Association for the Study of Diabetes/American Diabetes Association position statement, people with diabetes are first managed with diet/exercise, then oral agents, before finally progressing to injectable medications. ${ }^{2,3}$ Typically GLP-1 receptor agonists are the first injectable therapy that patients with T2DM are prescribed. ${ }^{2,3}$ In addition, participants with experience with injectable medications used to treat other conditions were excluded so that experience in other therapeutic areas would not influence their responses to the questions on their willingness to inject T2DM medications. After exposure to additional information on the injection devices, the participants did indicate an increased willingness to use injectable medications. At the beginning of the study, only $37.9 \%$ of the study participants expressed a willingness to take a treatment for T2DM that required an injection for each dose. After viewing the videos describing the delivery systems for the injectable medications, participants more frequently reported that they were willing to use injectable treatments.

The results of the current study should be interpreted with consideration for the following limitations. First, DCEs are a common methodology for examining preferences for attributes of a wide range of products and services. However, it is uncertain the degree to which the estimated preferences reflect the actual real-life medication decisions. Second, the sample population was predominantly male and White, and not markedly obese based on self-reported weight, which may limit the generalizability of the results. However, this reflected the proportion of males who indicated interest and were screened for the study, and is thus unlikely to reflect a bias based on the inclusion/exclusion criteria. Finally, as with all DCEs, the results of the study must be interpreted within the context of the attributes and levels that were included in the choice sets.

\section{Conclusion}

In this study, the attributes and levels presented to patients represented the actual safety and efficacy of dulaglutide and liraglutide observed in the only published head-to-head clinical trial to date (AWARD-6). ${ }^{15}$ The study provides information on patients' preferences for medication attributes that reflect the more subtle differences between two existing medications that are currently available as treatment options. The results of this study may help treatment providers better understand patients' preferences for the treatments examined in the context of this study, these are representative of decisions that they are likely to encounter in clinical practice. Specifically, the study highlights the potential importance of nonclinical factors such as dosing frequency and type of delivery system as important drivers of patient preference. This information may help guide patient-clinician treatment discussions, and facilitate a shared decision-making process focusing on each patient's specific needs and preferences. Finally, the results may provide more specific information on the factors and potential preferences of patients for dulaglutide versus liraglutide.

\section{Acknowledgments}

The authors would like to thank Fritz Hamme for his assistance in the preparation of this manuscript. This research was funded by Eli Lilly and Company.

\section{Disclosure}

HLG, JLP, and EWD are full-time employees of Evidera. $\mathrm{KSB}, \mathrm{SEC}$, and RP are full-time employees and minor shareholders of Eli Lilly and Company. The authors report no other conflicts of interest in this work.

\section{References}

1. Wild S, Roglic G, Green A, Sicree R, King H. Global prevalence of diabetes: estimates for the year 2000 and projections for 2030. Diabetes Care. 2004;27(5):1047-1053.

2. Inzucchi SE, Bergenstal RM, Buse JB, et al. Management of hyperglycemia in type 2 diabetes: a patient-centered approach: position statement of the American Diabetes Association (ADA) and the European Association for the Study of Diabetes (EASD). Diabetes Care. 2012;35(6): 1364-1379.

3. Inzucchi SE, Bergenstal RM, Buse JB, et al. Management of hyperglycemia in type 2 diabetes, 2015: a patient-centered approach: update to a position statement of the American Diabetes Association and the European Association for the Study of Diabetes. Diabetes Care. 2015 38(1):140-149.

4. Schwartz S, DeFronzo RA. The use of non-insulin anti-diabetic agents to improve glycemia without hypoglycemia in the hospital setting: focus on incretins. Curr Diab Rep. 2014;14(3):466.

5. Gallwitz B. Glucagon-like peptide-1-based therapies for the treatment of type 2 diabetes mellitus. Treat Endocrinol. 2005;4(6):361-370.

6. Polster M, Zanutto E, McDonald S, Conner C, Hammer M. A comparison of preferences for two GLP-1 products - liraglutide and exenatide - for the treatment of type 2 diabetes. J Med Econ. 2010;13(4):655-661.

7. Gelhorn HL, Stringer SM, Brooks A, et al. Preferences for medication attributes among patients with type 2 diabetes mellitus in the UK Diabetes Obes Metab. 2013;15(9):802-809.

8. Gelhorn HL, Stringer SM, Lee E, Palencia R. A discrete choice experiment conducted among patients with type 2 diabetes mellitus from the United States. Paper presented at: ISPOR 19th Annual International Meeting; May 31-June 4, 2014a; Montreal, QC, Canada.

9. Gelhorn HL, Stringer SM, Reinders S, Schreeb KH. German patients' preferences for attributes of type 2 diabetes medications. Paper presented at: ISPOR 17th Annual European Congress; November 8-12, 2014b; Amsterdam, the Netherlands. 
10. Mohamed AF, Zhang J, Johnson FR, et al. Avoidance of weight gain is important for oral type 2 diabetes treatments in Sweden and Germany: patient preferences. Diabetes Metab. 2013;39(5):397-403.

11. Hauber AB, Tunceli K, Yang JC, et al. A survey of patient preferences for oral antihyperglycemic therapy in patients with type 2 diabetes mellitus. Diabetes Ther. 2015;6(1):75-84.

12. Hauber AB, Nguyen H, Posner J, Ervin C, LaRue S, Kalsekar I. Patient preferences for frequency of glucagon-like peptide-1 receptor agonist (GLP-1RA) injections in the treatment of type 2 diabetes. Paper presented at: ISPOR 19th Annual International Meeting; May 31-June 4, 2014; Montreal, QC, Canada.

13. Bolen S, Wilson L, Vassy J, et al. Comparative effectiveness and safety of oral diabetes medications for adults with type 2 diabetes. Comparative effectiveness review No. 8 (Prepared by Johns Hopkins Evidence-based Practice Center under Contract No 290-02-0018). Rockville, MD: Agency for Healthcare Research and Quality; 2007.

14. Buse JB, Nauck M, Forst T, et al. Exenatide once weekly versus liraglutide once daily in patients with type 2 diabetes (DURATION-6): a randomised, open-label study. Lancet. 2013;381(9861):117-124.

15. Dungan KM, Povedano ST, Forst T, et al. Efficacy and safety of once weekly dulaglutide versus once daily liraglutide in type 2 diabetes (AWARD-6). Paper presented at: American Diabetes Association's 74th Scientific Sessions; June 13-17, 2014; San Francisco, CA.

16. Ahmann A, Szeinbach SL, Gill J, Traylor L, Garg SK. Comparing patient preferences and healthcare provider recommendations with the pen versus vial-and-syringe insulin delivery in patients with type 2 diabetes. Diabetes Technol Ther. 2014;16(2):76-83.

17. Boye KS, Matza LS, Walter KN, Van Brunt K, Palsgrove AC, Tynan A. Utilities and disutilities for attributes of injectable treatments for type 2 diabetes. Eur J Health Econ. 2011;12(3):219-230.

18. Jendle J, Torffvit O, Ridderstrale M, Ericsson A, Nilsen B, Bogelund M. Willingness to pay for diabetes drug therapy in type 2 diabetes patients: based on LEAD clinical programme results. J Med Econ. 2012; 15 Suppl 2:1-5.
19. Simon JA, Lewiecki EM, Smith ME, Petruschke RA, Wang L, Palmisano JJ. Patient preference for once-weekly alendronate $70 \mathrm{mg}$ versus once-daily alendronate $10 \mathrm{mg}$ : a multicenter, randomized, openlabel, crossover study. Clin Ther. 2002;24(11):1871-1886.

20. Silverman S, Calderon A, Kaw K, et al. Patient weighting of osteoporosis medication attributes across racial and ethnic groups: a study of osteoporosis medication preferences using conjoint analysis. Osteoporos Int. 2013;24(7):2067-2077.

21. Marshall D, Bridges JF, Hauber B, et al. Conjoint analysis applications in health - How are studies being designed and reported?: An update on current practice in the published literature between 2005 and 2008. Patient. 2010;3(4):249-256.

22. Instructions for Use: Trulicity ${ }^{\circledR} 1.5 \mathrm{mg}$ Single-Use Pre-filled Pen (Dulaglutide). Indianapolis, IN: Eli Lilly and Company; 2014.

23. Instructions for Use: Victoza $a^{\mathbb{B}} 6 \mathrm{mg} / \mathrm{mL}$ Solution for Injection in Pre-filled Pen (Liraglutide). Bagsvaerd, Denmark: Novo Nordisk A/S; 2013.

24. Orme B. Getting Started with Conjoint Analysis: Strategies for Product Design and Pricing Research. Madison, WI: Research Publishers, LLC; 2005.

25. Kuhfeld WF. Marketing research methods in SAS: experimental design, choice, conjoint, and graphical techniques. October 1, 2010. SAS 9.2 Edition. MR-2010. Available from: http://support.sas.com/techsup/ technote/mr2010.pdf. Accessed April 13, 2015.

26. Haymarket Media Group. MIMS. Available from: http://www.mims. co.uk. Accessed May 29, 2015.

27. Gelhorn HL, Stringer SM, Brooks A, et al. Patients' preferences for attributes of type 2 diabetes medications: a conjoint analysis. Paper presented at: ISPOR 15th Annual European Congress; November 3-7, 2012; Berlin, Germany.
Patient Preference and Adherence

\section{Publish your work in this journal}

Patient Preference and Adherence is an international, peer-reviewed, open access journal that focuses on the growing importance of patient preference and adherence throughout the therapeutic continuum. Patient satisfaction, acceptability, quality of life, compliance, persistence and their role in developing new therapeutic modalities and compounds to optimize

\section{Dovepress}

clinical outcomes for existing disease states are major areas of interest for the journal. This journal has been accepted for indexing on PubMed Central. The manuscript management system is completely online and includes a very quick and fair peer-review system, which is all easy to use. Visit http://www. dovepress.com/testimonials.php to read real quotes from published authors. 